\title{
Resultados, Aprendizajes y Perspectivas de la Plataforma PEPE como Contribución a la Educación Escolar
}

\author{
Results, Learning, and Perspectives of the PEPE Platform as a Contribution to School \\ Education
Resultados, Aprendizagens e Perspectiva da Plataforma PEPE como Contribuição para a Educação Escolar

\author{
Roberto Canales R., ${ }^{\mathrm{a}}$ Martín Quintana E., ${ }^{\mathrm{b}}$ Cristina Alarcón S., ${ }^{\mathrm{c}}$ Andrea Hain C., ${ }^{\mathrm{d}}$ \\ Macarena Gárnica A. ${ }^{\mathrm{e}}$
}

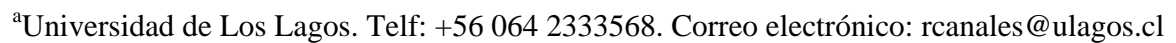 \\ bUniversidad de Los Lagos. Telf: +56 064 2333315. Correo electrónico: m.quintana@ulagos.cl \\ ${ }^{c}$ Universidad de Los Lagos. Telf: +56 064 2333237. Correo electrónico: calarcon@ulagos.cl \\ ${ }^{\mathrm{d}}$ Investigadora independiente. Telf: +56 064 2333053. Correo electrónico: hain.andrea@gmail.com \\ ${ }^{\mathrm{e} U n i v e r s i d a d ~ d e ~ L o s ~ L a g o s . ~ T e l f: ~+56 ~} 064$ 23333074. Correo electrónico: macarena.garnica@ulagos.cl
}

\begin{abstract}
RESUMEN
Se presenta un informe sumario y crítico que da cuenta del proceso y resultados del proyecto Rompiendo la brecha digital para un uso significativo de las TIC en establecimientos educacionales: Plataforma de Entornos Pedagógicos Especializados (PEPE), ejecutado entre 2009 y 2011 principalmente en la región de los Lagos, Chile. A partir de resultados empíricos que evidencian mejoras en niveles de desempeño de estudiantes, en lenguaje y matemática, se moviliza el foco de trabajo desde una plataforma centrada en contenidos, al proceso de colaboración en una comunidad de aprendizajes; en definitiva la transición epistémica está dada por el avance desde lo individual a lo colectivo. Esta transición no puede sino comprenderse como signo de actualidad en una sociedad que se interroga sobre la educación en tiempos de crisis.
\end{abstract}

Palabras claves: plataforma virtual, comunidad de aprendizaje, educación y TIC.

\begin{abstract}
Presented here is a summarized and critical report which discusses the process and results of the Project "Rompiendo la brecha digital para un uso significativo de las TIC en establecimientos educacionales: Plataforma de Entornos Pedagógicos Especializados" (PEPE). The Project was executed between 2009 and 2011, primarily in Los Lagos region, Chile. With the empiric results that evidence improvements in the levels of students' performance, in language and mathematics, the focus is mobilized from a platform centered on contents, to the process of collaboration in a community of learning. On the whole, the epistemic transition is given by the advancement from the individual to the collective. This transition cannot be understood except as a sign of contemporary in a society that interrogates itself about education in times of crisis.
\end{abstract}

Keywords: virtual Platform, learning community, education and ICT.

\footnotetext{
* Financiado por el IV concurso TIC-EDU de Fondef Conicyt de Chile, ejecutado entre los años 2009 y 2011. Institución principal Universidad de Los Lagos y secundaria Universidad de Chile. Los socios del proyecto fueron el DAEM de Osorno y la Red Universitaria Nacional REUNA.
} 


\section{RESUMO}

Apresentamos o relatório resumido e crítico do Projeto "Rompiendo la brecha digital para un uso significativo de las TIC en establecimientos educacionales: Plataforma de Entornos Pedagógicos Especializados (PEPE)", executado entre 2009 e 2011, principalmente na região de Los Lagos, no Chile. A partir dos resultados empíricos, que mostram melhorias nos níveis de aproveitamento dos estudantes em relação à linguagem e à matemática, enfatizamos o estudo desde uma plataforma centrada em conteúdo ao processo colaborativo em uma comunidade de aprendizagem. Finalmente, a transição epistemológica é dada pelo avanço que segue desde o individual até o coletivo. Essa transição somente pode ser compreendida como um sinal da atualidade, em uma sociedade que indaga questões sobre a educação em tempos de crise.

Palavras chave: plataforma virtual, comunidade de aprendizagem, educação e tecnologias.

\section{EL PROBLEMA DE ORIGEN: ACTUALIDAD Y CONTEXTOS}

El presente artículo se constituye en una revisión crítica del proyecto Rompiendo la brecha digital para un uso significativo de las TIC en establecimientos educacionales: Plataforma de Entornos Pedagógicos Especializados, PEPE, financiado por el Fondo de fomento al desarrollo científico y tecnológico de Chile, a través de su IV concurso Tic-Edu el año 2008, y ejecutado por un consorcio liderado por la Universidad de Los Lagos entre el año 2009 y el 2011. ${ }^{\circledR}$

El texto en general se organiza en tres acápites. En primer lugar, se presenta una síntesis del problema que dio origen a la propuesta y algunos elementos constitutivos de su reconsideración de actualidad; en segundo lugar, se presentan y describen los resultados y; en tercer lugar, se analizan los aprendizajes y perspectivas.

La concepción y ejecución de un proyecto como PEPE, demanda la imbricación de dos tipos de análisis. El primero tiene relación con las promesas investigativas específicas implicadas en su diseño, a partir de las cuales es posible reconocer el valor que finalmente aportó esta iniciativa; la puesta en valor de dichos resultados dependerá, en todo caso, de la capacidad que tuvo de confrontar su tiempo presente, solo hurgado en función de un contexto y estado del arte que a la luz de otro presente, debe ser movilizado. Ello será parte constitutiva del acápite de los resultados del proyecto PEPE. El segundo análisis parte de la pregunta ¿cómo avanzar en la investigación? Parafraseando al etnógrafo Gertz (1997), la investigación no se hace sola. Ello implica que tras la identificación del problema, la definición de los dispositivos teórico metodológicos que contribuyan a esbozar respuestas posibles, hay sujetos, equipos humanos que se cuestionan desde un contexto determinado, donde problemáticas e investigadores resultan, a la postre, parte de una misma narrativa epistémica que propende, en definitiva, a la producción de un conocimiento que se releva como oportunidad de mejoramiento a las condiciones de nuestro estar en el mundo. En razón de dicha condición es que la distancia fenomenológica se hace necesaria. Observar aquello que ha quedado ya en el horizonte de lo hecho, desde una mirada crítica, permite levantar algunos antecedentes que impactan sobre el fenómeno mismo, pero sobre todo en los sujetos que aprenden; ello será preocupación del acápite Aprendizajes y perspectivas del proyecto PEPE.

\footnotetext{
${ }^{1}$ La Universidad de Chile fue institución co-ejecutora. Miembros del consorcio como socios fueron, igualmente, la Ilustre Municipalidad de Osorno, a través de su Departamento de Educación Municipal y la Red Universitaria Nacional REUNA.
} 
El año 2008, tres escenarios fueron los fundamentos del diseño del proyecto PEPE. En primer lugar, la medición estandarizada de los resultados educativos en ciertas disciplinas y niveles (lenguaje y matemática en el $\mathrm{SIMCE}^{2}$ ) se mostraban magros. Tan solo como ejemplo, a partir de los resultados del año 2007 en lectura, un $27 \%$ de los estudiantes evaluados se ubicaban en el Nivel Intermedio, un $33 \%$ en el Nivel Avanzado y más de un $40 \%$ en la categoría Inicial (Mineduc, 2008). De otra parte, en aquellas pruebas estandarizadas en que Chile se sometía a comparación con otros países, la situación era similar. En la prueba PISA $^{3}$, por ejemplo, pese a que los promedios obtenidos el año 2006 en Lectura, mostraban una mejora significativa en relación a los resultados obtenidos el 2000, e incluso Chile era el país que más aumentaba su puntaje en (33 puntos en lectura) entre 32 países. Lo cierto es que un porcentaje cercano al $55 \%$ de estudiantes chilenos estaban en el nivel 1 y bajo 1 , los peores del ranking.

Este es un dato preocupante, porque más de la mitad de los estudiantes en Chile no han desarrollado competencias que les permitan enfrentar situaciones problemáticas de vida que impliquen el uso de las matemáticas. Su razonamiento matemático sólo se aplica a contextos muy familiares; podrían usar procedimientos rutinarios, siguiendo instrucciones y no siendo capaces de proponer alternativas de resolución de problemas. [...] El porcentaje de estudiantes chilenos bajo el nivel 1 era tres veces el del promedio de la OCDE y el porcentaje en el nivel 1 fue casi el doble de ese promedio. Por el contrario, el porcentaje en los niveles 5 y 6 en la OCDE fue tres veces el que existe en Chile (Brunner, 2007:10)

Entonces, abordar un desafío propositivo orientado a contribuir con mejoras en la educación chilena, no podía soslayar que la problemática nacional referida al desempeño escolar se materializaba en la persistencia de problemas de lectura y escritura, además de una sostenida deficiencia en matemática, especialmente en estudiantes de sectores vulnerables.

En segundo lugar, se evidenciaban, igualmente, brechas de segregación social importantes entre establecimientos, cuestión que se reflejaba en que los resultados educativos eran aún peores en aquellos establecimientos de dependencia municipal o particulares subvencionados ${ }^{4}$. Por ejemplo, en los resultados SIMCE 2006 ya referidos más arriba, el $40 \%$ de los estudiantes que obtuvo nivel inicial se encontraba en el segmento bajo y medio bajo de los grupos socioeconómicos del país.

En tercer lugar, había necesidad de fortalecer el uso apropiado de las tecnologías a nivel escolar. El informe de Desarrollo Humano en Chile, elaborado por el PNUD el año 2006 y cuyo foco estuvo en las TIC, señalaba, por ejemplo, que las principales dificultades no estaban en el acceso a ellas, sino más bien en las significaciones de su uso, temática que evidenciaba una debilidad en las estrategias y modelos pedagógicos

\footnotetext{
${ }^{2}$ Sistema de Medición de la Calidad y Equidad. Se trata de una prueba estandarizada que se aplica a ciertos niveles y disciplinas del sistema escolar chileno. Su diseño e implementación depende del Ministerio de Educación de Chile.

${ }^{3}$ El Programa Internacional de Evaluación de Estudiantes (PISA, por sus siglas en inglés) es una medición que desarrolla la OECD, para la evaluación de competencias en lectura, escritura, matemática y ciencias a estudiantes de 15 años de más de 70 países del mundo. Para más información remitirse a http://www.oecd.org/pisa/

${ }^{4}$ Tres tipos de dependencia tienen los establecimientos educacionales en Chile, los municipales con financiamiento público, los particulares subvencionados con financiamiento compartido público y privado, y los particulares pagados.
} 
de implementación. Así, uno de los grandes desafíos era cambiar el foco de atención desde el uso tradicional de estas tecnologías a otro que resignificaba la condición del sujeto en la Sociedad de la Información y el Conocimiento. De ese modo, a la luz del análisis de las orientaciones del PNUD (2006) y las políticas públicas nacionales, se le da continuidad al programa Enlaces del Ministerio de Educación de Chile; el Plan Tecnologías para una Educación de Calidad, es el instrumento con que el Ministerio de educación actualizó su política en educación y TIC, e inyectó recursos para fortalecer el equipamiento tecnológico, promoviendo además la generación de planes complementarios, por ejemplo TIC en el Aula, que precisamente concentraban su esfuerzo en la facilitación de condiciones materiales y no materiales para el buen uso pedagógico de los recursos tecnológicos instalados.

En este sentido, algunas cuestiones del contexto global y local que intervienen hoy en la lectura de lo que ha pasado desde entonces y que habría que tener en consideración son:

- En el 2010, Chile entra de lleno a la OCDE con plenos derechos y deberes. El estándar en educación deja de ser solo interno y se acelera la necesidad de compararse con el mundo.

- El desarrollo de la industria tecnológica no solo se ha masificado, sino que además se ha diversificado con la aparición y masificación de smartphone y tablet. Rinaldi (2011), a partir de datos del informe Horizon 2011, señala que al 2009 el 30\% de los dispositivos telefónicos vendidos ya contaban con tecnología smarthphone; se proyectaba que para el 2013 esa cifra crecería a cerca del 80\%, logrando un acceso de 4 mil millones de usuarios en el mundo. El concepto de ubicuidad se impone como preocupación central y una oportunidad sin precedentes para la educación.

- Hay dos avances significativos en cuanto a disposición de información sobre educación y tecnología en Chile: el 2009 se realiza el Censo de Informática Educativa, coordinado por Enlaces. Los resultados son elocuentes, la región de Los Lagos es la peor evaluada del país con un Índice de Desarrollo Digital Escolar $^{5}$ (IDDE) de 0,42 (Enlaces, 2013), cuestión que se ratifica en el indicador 'Uso de TIC' donde la región obtiene un 0,46 (la más baja del país) frente al 0,58 nacional. El indicador 'horas que el profesor usa TIC con alumnos', muestra que en la región hay un uso sustantivamente mayor que en el resto del país (un 5,24 hrs. respecto del promedio nacional 4,88 hrs.) Ello pudiera indicar que el uso no está siendo eficiente, pues dicho tiempo no se refleja en el IDDE general de la región. Contrastar este dato con los resultados de la evaluación docente es ilustrativo (MINEDUC, 2011). Allí se declara que un 79\% de la muestra de docentes utiliza el computador todos los días; dichos usos se agrupan en búsqueda de información y preparación de material; sólo un 30\% del uso se dedica a compartir con otros profesores. A partir del 2011 se instala la Medición SIMCE TIC $^{6}$ y si bien se relativiza la débil condición regional, no deja de ser preocupante que a nivel nacional el 50,5\% de los estudiantes obtenga solo un nivel inicial (de 3 niveles: inicial, intermedio y avanzado). La Región de Los Lagos, si bien no es la peor, por los 243 puntos obtenidos, se ubica igualmente dentro del segmento inicial; más del $90 \%$ se encuentra en el segmento inicial e

\footnotetext{
${ }^{5}$ Refleja el grado en que las escuelas han desarrollado las condiciones de infraestructura, capacidades humanas, gestión y uso de Tecnologías de Información y Comunicaciones (TIC) http://www.enlaces.cl

6 Evalúa 12 Habilidades TIC para el aprendizaje, agrupadas en tres dimensiones: Información, comunicación y ética e impacto social. http://www.enlaces.cl
} 
intermedio (ENLACES, 2013).

- Cifras más cifras menos, se observa desde el 2009 un marginal repunte de los resultados en pruebas estandarizadas, fundamentalmente en matemática. En efecto, los resultados SIMCE 2011 permiten constatar que, al menos en lenguaje, los estudiantes de los sectores más vulnerables han logrado un sostenido avance respecto de mediciones anteriores, sin embargo, en los resultados 2012 se observa una baja de 3 puntos (baja de 249 a 246); en los demás segmentos prácticamente no hay diferencias. La situación en matemática para este nivel es un poco mejor, notándose de todas formas un estancamiento o variaciones que el propio SIMCE considera no significativas. Para segundo medio, la situación es levemente distinta, en la prueba de lenguaje todos los sectores socioeconómicos bajan o no presentan variación, salvo la baja sustantiva en el segmento alto. En matemática, sin embargo, hubo un repunte significativo y generalizado.

Es, precisamente, desde estas condiciones de contexto que debe evaluarse la contribución de PEPE a la educación chilena. A continuación una exposición sumaria de sus principales resultados.

\section{RESULTADOS DEL PROYECTO PEPE}

El proyecto PEPE, tuvo como propósito general mejorar el desempeño escolar de los estudiantes de establecimientos educativos, especialmente municipales, mediante el acceso a una plataforma que articula recursos digitales y objetos de aprendizaje, orientada a las necesidades de una comunidad educativa que aprende, en las áreas de matemática y lenguaje y comunicación para los niveles parvulario y primer ciclo básico ${ }^{7}$.

En el año 2009 se diseñó y desarrolló la red PEPE, en el 2010 se implementó a nivel piloto en diez establecimientos educacionales de dependencia municipal de la comuna de Osorno, mientras la etapa de masificación o escalamiento se desarrolló en el 2011, instancia en la que se sumaron veinte escuelas de la región de Los Lagos y de la Araucanía, además de tres escuelas de la ciudad de Pereira en Colombia, beneficiando a 10.000 estudiantes y cerca de 300 docentes aproximadamente.

Los establecimientos focalizados en la etapa piloto atendieron a estudiantes de similares condiciones socioeconómicas correspondientes a rango medio, medio bajo y bajo. Para esta clasificación se consideraron tres variables: nivel educacional de los padres, ingreso familiar mensual e índice de vulnerabilidad, cuya información fue proporcionada por el Departamento de Administración Educacional Municipal DAEM de Osorno.

Se definió una muestra no probabilística intencionada, conformada por diez escuelas básicas municipales de la Ciudad de Osorno. Se consideraron los siguientes criterios de selección muestral: i) establecimiento educativo de dependencia municipal; ii) establecimiento educativo vinculado al programa Enlaces; iii) establecimiento educativo que imparta educación parvularia y básica y; iv) establecimiento educativo que cuente al menos con dos cursos por nivel desde $2^{\circ}$ nivel de transición a $4^{\circ}$ año básico. En definitiva, la muestra para la realización del piloto del proyecto consideró a 450 estudiantes, es decir, 5 estudiantes por nivel desde transición II del nivel parvulario

\footnotetext{
${ }^{7}$ El nivel parvulario en Chile atiende a niños y niñas de 0 a 5 años de edad, y el primer ciclo básico comprende el rango de 6 a 9 años aproximadamente.
} 
al cuarto año básico, de los 10 establecimientos educacionales.

Desde una mirada tecnológica, cada establecimiento educacional adherido a la red contó con un portal web institucional personalizado y multipropósito, en el que de manera autónoma y descentralizada se publicaban noticias y fotografías de la comunidad escolar e información relativa al equipo de gestión directivo y de la unidad técnica pedagógica, desplegando información relacionada con talleres educativos, reglamentos, temáticas del Centro de Alumnos, Centro de Padres, etc. Adicional a la web institucional, se instaló en cada escuela un aula virtual a la cual ingresaban diariamente estudiantes y profesores. Ésta contenía aplicaciones educativas interactivas, tales como objetos de aprendizaje, ejercicios en línea, animaciones, enlaces a web, videos y presentaciones, todo asociado a los aprendizajes esperados que determinaba el Ministerio de Educación de Chile para las áreas matemática y lenguaje y comunicación. Desde una perspectiva pedagógica, se trabajó en fortalecer las competencias tecnopedagógicas de los profesores y de los encargados técnicos de cada establecimiento a través de talleres de asesoría pedagógica directa, capacitación y acompañamiento en el aula, entregando, en cada ocasión, manuales de usuarios del sistema y promoviendo el uso de estrategias didácticas contextualizadas para la implementación de PEPE en la sala de clases.

PEPE asumió cuatro principios claves para abordar las tecnologías en el sistema educativo: la simplicidad de uso, la facilidad en la administración, la robustez del sistema y el compromiso con el aprendizaje. La simplicidad de uso como principio se refleja en la instalación y el entorno virtual de aprendizaje asociado de manera directa y sencilla al aula virtual y los recursos, tanto para profesores como para estudiantes. En este sentido, el profesorado normalmente no integra las tecnologías en la enseñanza por las dificultades que presentan y la inseguridad de perder el control tecnológico en la sala de clases. PEPE, además, es muy simple de administrar; los docentes recibieron una formación inicial para aprender a subir las noticias de la comunidad educativa y actualizar la información del portal institucional del establecimiento, asimismo, aprendieron a trabajar en el aula virtual, la que requiere de indicaciones básicas que rápidamente se asimilan y logran niveles de autonomía para incorporar nuevos recursos, para actualizarlos y/o eliminarlos. Pepe es robusto como sistema. Los servidores dedicados para la administración y su red de establecimientos educacionales cumplen altos estándares de seguridad, de hecho están alojados en la sala de servidores de la Universidad de Los Lagos, pero el uso es exclusivo de las escuelas que se conectan a la red, lo que implica que el sistema se mantiene funcionando las 24 horas del día y los 365 días del año. Finalmente, el último principio es el compromiso con el aprendizaje. El foco de atención está instalado en el aprendizaje de los estudiantes, en este sentido, el desarrollo tecnológico de cada una de las aplicaciones ha estado al servicio docente en su labor de enseñar, pero en especial al estudiante en su actividad de aprender, asociando el desarrollo de los recursos digitales a los aprendizajes esperados definidos por el Ministerio de Educación de Chile (Canales et al. 2012).

La evaluación en torno a la implementación de la Plataforma PEPE se realizó en relación a los efectos en el desempeño escolar de los estudiantes en las áreas de matemática y lenguaje y comunicación, y en torno a la satisfacción de los docentes frente al desempeño escolar de sus estudiantes al utilizar PEPE como herramienta de apoyo al proceso de enseñanza y aprendizaje. Desde una perspectiva metodológica investigativa, se trabajó con un enfoque mixto. Lo cuantitativo a través de un diseño cuasiexperimental: un grupo control y otro experimental, con foco en el análisis de desempeño de los estudiantes y el uso de la plataforma tecnológica PEPE.

Las hipótesis del estudio fueron dos, la primera dice relación con que los 
estudiantes que utilizan la Plataforma PEPE en las áreas de matemática y lenguaje y comunicación evidencian un mejor desempeño escolar que aquellos que no la usan; la segunda señala que los profesores que utilizan la Plataforma PEPE durante el proceso de enseñanza evidencian altos niveles de satisfacción en relación al desempeño de sus estudiantes.

A nivel conceptual se entenderá por desempeño escolar la conducta motivada frente al aprendizaje y el nivel de conocimiento demostrado en el proceso de enseñanza y aprendizaje. Para evaluar dicha conducta se realizó un cuestionario tipo Likert para recoger la percepción de los estudiantes, mientras que para el caso del nivel de conocimiento se aplicó una prueba de contenido pre y post test al grupo control y experimental. El componente cualitativo se abordó a través de una entrevista semiestructurada que recoge la satisfacción percibida por los docentes en torno al nivel de aprendizaje y la conducta motivada de los estudiantes al utilizar PEPE como herramienta de apoyo al proceso de enseñanza y aprendizaje.

En términos de resultados, se concluye que "los estudiantes que utilizaron la plataforma PEPE y sus recursos digitales de aprendizaje en matemática, por un periodo de 5 meses y sobre 12 sesiones de trabajo, mejoraron un 10,1\% sus aprendizajes con respecto a aquellos estudiantes que no la usaron" (Canales et al. 2012: 47). Por otro lado, al promediar los niveles de logro comparados con los grupos control de todos los niveles educativos, se puede señalar que los estudiantes del primer ciclo básico, considerando el nivel de transición mayor al cuarto año básico, que utilizaron la plataforma PEPE en forma sistemática en un periodo de 5 meses, mejoraron sus aprendizajes en lenguaje y comunicación en un $6,12 \%$ en comparación a los niveles de avance de aquellos estudiantes que no usaron la plataforma de entornos pedagógicos especializados PEPE (Canales et al. 2012).

De la entrevista semiestructurada que se aplicó a una muestra de 9 profesores de una población de 50, se evidencia en relación a la satisfacción frente al desempeño escolar de los estudiantes que:

existe una alta satisfacción de los docentes en torno a la conducta motivada de los estudiantes al trabajar con estos recursos como apoyo a las clases de matemática y lenguaje y comunicación, señalando por ejemplo en distintas variables consultadas altos niveles de compromiso de los estudiantes por desarrollar las tareas, alta participación en el desarrollo de la clase y alta persistencia en resolver los problemas asociados a las tarea. (Canales et al. 2012:47)

Respecto de la variable motivación, relacionada al compromiso de los estudiantes por aprender, los profesores señalan que los estudiantes al trabajar con PEPE realizan sus tareas inmediatamente después de que se les entregan las instrucciones, terminan a tiempo las actividades trabajadas en clases, hacen bien las actividades y se concentran más fácilmente durante el desarrollo de las mismas. Sobre la misma variable, pero esta vez relacionada a la participación de los estudiantes en el proceso educativo, los profesores consultados plantean que al utilizar PEPE los estudiantes participan mucho más, ya que escuchan con atención las explicaciones, responden a las preguntas que se les hacen, se ayudan y conversan con sus compañeros y compañeras sobre la actividad en la cual deben trabajar, asimismo, al presentar dudas piden ayuda al profesor o compañero.

Finalmente, desde la variable motivación relacionada a la persistencia de los estudiantes para desarrollar el trabajo, los profesores indican que los estudiantes se distraen menos al realizar una tarea utilizando PEPE, pues cuando tienen un problema para realizar la tarea intentan solucionarlo hasta que pueden terminarla. Asimismo, 
opinan que a sus estudiantes les cuesta menos empezar a trabajar la tarea cuando utilizan el computador, ya que trabajan diligentemente, se muestran más entusiastas y sienten que el tiempo pasa más rápido.

En síntesis, los profesores plantean que la plataforma PEPE les facilita el trabajo especializado por cada una de las disciplinas, en este sentido comentan lo positivo de

tener esa facilidad de poder ingresar a cualquier objeto, de acuerdo a tu nivel [948],

... era mucho más fácil para nosotros ingresar al PEPE y ya sabíamos que teníamos los objetos de aprendizaje, entonces ya uno planificaba de acuerdo a eso [1340:1341]

para uno como profesor se le facilita bastante el trabajo, ya no estaba sólo dedicada a buscar material [1354:1355].

Otro docente planteaba

... A mí me facilitó mucho el trabajo, porque yo tendría que haber elaborado mucho material concreto, en cambio el hecho que esté en la plataforma todo, el objeto ahí, a mi me alivianó mucho el trabajo [1303:1305].

Los docentes señalaban que para ellos es una excelente herramienta de apoyo a la didáctica de la clase

también me sirvió como idea para hacer guías, para preparar mis clases, entonces lo trabajé con los alumnos y también lo trabajé individualmente para mí [1487:1489].

Plantean que el uso de la Plataforma les permite integrar a la familia al proceso educativo indicando

los padres estaban muy contentos, contentos de que este curso había participado en el proyecto [1575:1576].

Agregaban los profesores

me contaban los papás lo que habían hecho, por eso yo sabía que sí habian entrado [1582:1583].

Los resultados en relación al impacto social indican que el proyecto PEPE como innovación didáctica ha logrado impactar en distintos contextos y actores, tal es el caso que en la formación inicial docente se logró articular su accionar con el eje de práctica profesional, a partir de la cual estudiantes universitarios, futuros profesores, integraban curricularmente las tecnologías utilizando los recursos que proveía PEPE.

Así como fue importante en la articulación con las prácticas pedagógicas, también lo fue en la formación de los estudiantes del área pedagógica, tanto de pregrado como de postgrado, quienes en sus asignaturas asimilaban el modelo de PEPE y las estrategias de uso, para luego volcarlas a su quehacer pedagógico en la sala de clases.

PEPE colaboraba con el docente y con los estudiantes, permitía que estudiantes 
más aventajados adelanten los contenidos revisando recursos digitales de cursos y niveles superiores, mientras aquellos estudiantes que tenían dificultades de aprendizaje podían repasar los contenidos tanto en la sala de clases como en los hogares.

A medida que el proyecto se instalaba en distintos establecimientos, los requerimientos iban en aumento, tal como sucedió en los sectores rurales donde no había conexión a Internet, allí se hizo necesario desarrollar un PEPE Portable, que posibilitó tanto la instalación plataforma institucional de la escuela, como el aula virtual y los objetos de aprendizaje a través de un dispositivo de almacenamiento externo de 600 megabytes tipo pendrive conectado directamente a los computadores personales o, en su defecto, a una red local; esto facilitaba el acceso de todos al uso de las tecnologías para aprender.

Dentro de las condiciones de éxito del proyecto PEPE, destacan la coordinación e implicancia del equipo investigador con la dirección y autoridades escolares, la coordinación con los encargados pedagógicos en las escuelas, la coordinación con docentes o profesionales encargados de las TIC comunales y escolares, la capacidad de intencionar el uso de las TIC para el aprendizaje en el programa educativo institucional de la escuela y la planificación curricular que realizaban los profesores con la integración de PEPE, además del acompañamiento a los docentes en el proceso educativo.

Por último, fueron varias las estrategias que se implementaron para tener éxito en la incorporación de PEPE en las escuelas municipales, entre ellas la socialización con las autoridades políticas y académicas, sostenedores, alcalde y concejo municipal, directores de DAEM y de colegios, jefes de la unidades técnicas pedagógicas y profesores. A través de charlas o talleres se exponían las virtudes del programa para que apoyen, desde la política pública, su inclusión en la sala de clases. Es relevante destacar el acompañamiento que el equipo de investigadores realizaba en cada una de las escuelas, sobre todo al principiar la implementación de la iniciativa, instancia en que el profesor se sentía inseguro, pero que, luego de conocer aspectos básicos del sistema, empezaba a trabajar en forma autónoma.

Igualmente, es importante consignar un dato: sobre el 20\% de las navegaciones en la plataforma PEPE se realizaba desde los hogares de los estudiantes, esto refleja que las tecnologías estaban siendo utilizadas no sólo en la escuela, sino también en los hogares y para estudiar, situación que fue confirmada tanto por los profesores como por los padres y apoderados.

\section{APRENDIZAJES Y PERSPECTIVAS}

Después de cuatro años de funcionamiento del proyecto PEPE, se detectan distintos niveles de aprendizajes, oportunidades de mejora y de sostenibilidad en el tiempo de la plataforma.

Desde el punto de vista de los aprendizajes, si bien es cierto los estudiantes ejercitan con los recursos pedagógicos que están a su disposición, los profesores no tienen un control de los niveles de avance individual ni grupal de los aprendizajes de los estudiantes en términos de conocimientos, ya que PEPE no reporta esta información, limitando una estrategia clave en el modelo didáctico que es la retroalimentación y la evaluación continua del proceso de enseñanza, por un lado, y del aprendizaje, por otro. Desde el punto de vista relacional, no se posibilitó la instalación de una comunidad de aprendizaje autónoma que permita la interacción e intercambio de experiencias entre los profesores que hacen uso del sistema, lo que ha redundado en la escasa producción de 
recursos digitales por parte de los docentes, y por ende la baja posibilidad de compartir experiencias de uso de las tecnologías en la sala de clases. Cabe considerar que no era el sentido inicial del proyecto, pero hoy se percibe como una necesidad.

Desde el punto de vista tecnológico y considerando el soporte, PEPE fue diseñado y desarrollado como un sistema multiplataforma, disponible para funcionar prácticamente en cualquier tipo de computador, sin embargo, no se consideró su uso en dispositivos móviles, como tablets o smartphones, lo que sin lugar a dudas va en desmedro de la portabilidad y accesibilidad del sistema.

En otro sentido, en términos de los accesos al sistema, éstos en PEPE se desarrollaron bajo la modalidad SSO (Single Sign On), lo que quiere decir que cada usuario que se autentifica al ingresar a uno de los subsistemas, automáticamente lo hace en el sistema local completo (portal PEPE, portal de la escuela, aula virtual de la escuela y repositorio compartido). En relación a los perfiles de usuario, las acciones y atributos al interior de las aulas virtuales es limitada, debiendo remitirse a un uso más bien básico de los servicios provistos, sin poder interactuar entre los actores del sistema, tales como jefes de UTP, profesores, estudiantes, padres y apoderados.

Por último, el sistema de almacenamiento, búsqueda y clasificación de los nuevos recursos del repositorio actual, a pesar de estar integrado al sistema PEPE, adolece de la facilidad y flexibilidad de uso, temática que ha conspirado con el mejor aprovechamiento de este servicio.

Partiendo de esta descripción, el análisis crítico de la ejecución del proyecto PEPE comienza por generar un espacio de reflexión sobre la posibilidad de construir una plataforma de metacuestionamiento, que eventualmente podría generar nuevos focos en el equipo, oxigenar perspectivas y sostener decisiones que pudieran dar respuesta a la interrogante respecto de cómo continuar. Esta etapa en su comienzo fue mediada por una técnica tan sencilla como popular: el análisis FODA descrito a continuación:

Fortalezas. La robustez técnica y política del dispositivo y del equipo de investigación, consolidado en programas académicos de educación continua y postgrado, además de una capacidad productiva y de fortalecimiento de masa crítica, en desarrollo.

Debilidades. A pesar del esfuerzo de búsqueda, desarrollo y catalogación por disponer de un repositorio amplio y diverso de recursos de aprendizaje, se observa una limitación ontológica. Nunca el repositorio será, en realidad, lo suficientemente nutrido si éste no se construye de manera conjunta con los docentes. Esta condición supone superar una dificultad en el sentido de apropiación del dispositivo por parte de los docentes. La facilitación de condiciones para dicha apropiación implica, entonces, la ruptura con los niveles y la flexibilización de la disciplina. En efecto, no puede el mismo sistema generar obstáculos para la participación.

Oportunidades. La situación de la educación chilena y el modelo social y político que la sostiene se encuentra en crisis. Ello si bien constituye una amenaza a los sujetos que habitan este territorio, en realidad es igualmente una oportunidad, un campo fértil para la creatividad y la propuesta. Cuando las cosas marchan de acuerdo a lo planeado y hay un meridiano acuerdo social respecto de que están bien, entonces el espacio para el cambio se restringe a la reforma, a los maquillajes, a los ajustes. Cuando no, entonces es el tiempo de las propuestas y la participación. La mirada en lo local se constituye así en oportunidad de impacto y apropiación plausible.

Amenazas. Fundamentalmente el avance de la informática educativa ha debido convivir con su principal amenaza: suponer que será la tecnología la encargada de superar la crisis, de mejorar aprendizajes y, en definitiva, de mejorar la calidad de la 
educación. La tecnología se vuelve y se confronta hacia sí misma, constituyéndose entonces en algo como aquello que Baudrillard denomina un simulacro, "disimular es fingir no tener lo que se tiene. Simular es fingir tener lo que no se tiene. Lo uno remite a una presencia, lo otro a una ausencia" (Baudrillard, 2007: 6). Estas cuatro condiciones de reflexividad permiten establecer el piso para una lectura de los cambios más significativos en la realidad que fue abordada por PEPE y la que se confronta hoy.

En consideración del análisis descrito, uno de los aspectos relevantes que quedó en evidencia con la ejecución del proyecto PEPE es la participación de los docentes en el intercambio de sus prácticas. La no satisfacción de la necesidad social de favorecer intercambios y aprendizajes socialmente elaborados, contemplando de manera inclusiva la co-participación entre docentes de establecimientos de diferentes realidades sociales y culturales, aporta igualmente a uno de los "cuellos de botella" del modelo educativo chileno: la segregación (Valenzuela et al., 2010). En ese contexto, si consideramos que las Tecnologías de la Información y las Comunicaciones (TIC), de la cual la plataforma PEPE es parte, se entienden como una producción y reproducción de símbolos de información y conocimiento; tecnologías que construyen nuevos espacios/tiempo, donde se constituyen diversos perfiles de relación social, nuevas formas institucionales y nuevas categorías de aprehensión de la experiencia personal y social (Vizer, 2003), en concordancia con Castell (1999, en Peña, 2002), lo que haría relevante a las tecnologías, sería la capacidad de penetración en distintos sectores y actores de la sociedad, con potenciales efectos socioculturales y económicos; que tienen una lógica de interconexión de todo el sistema o conjunto de relaciones dentro y fuera de éste. Es decir, deberían potenciar la transformación en el proceso de enseñanza aprendizaje (EA), crear nuevos entornos de E-A donde la interconexión produciría una relación reciproca entre los usuarios (Coll y Bustos, 2010).

Se hace necesario, entonces, a modo de aprendizaje, la necesidad de sistematizar practicas con un claro foco en la noción de comunidad de aprendizaje utilizando TIC, ya que ésta se constituiría en un lugar donde interactúan

actividades de enseñanza y aprendizaje de calidad, donde intervienen profesores, estudiantes y las propias tecnologías mediante prácticas que resultan eficientes y eficaces, eficientes porque aportan ventajas que otros medios no nos aportan, y eficaces porque logran los objetivos educativos, es decir mejorar los aprendizajes (Canales y Marqués, 2007).

Esto remite a la idea de inclusión de un grupo de personas con diferentes niveles de experiencia, conocimiento y pericia, quienes aprenden gracias a la colaboración que establecen entre sí, a la construcción del conocimiento colectivo que llevan a cabo y a los diversos tipos de ayuda que se prestan mutuamente (Coll y Bustos, 2010). De esta forma, la comunidad se convierte en un medio entre los sistemas socio-técnicos y las prácticas socio-culturales de difusión y almacenamiento de la información, que sirve para la configuración de la comunicación/interacción y que determina las percepciones y la formación de experiencias, tanto colectivas como individuales, hecho que se puede expresar hipermedialmente a través de variadas aplicaciones, documentos, enlaces, vínculos, sonido, gráfico, video y con diversos protocolos de transmisión de datos.

Otro aprendizaje que se desprende de la implementación de PEPE es la necesidad del desarrollo de una usabilidad social de la plataforma, definida por la vinculación y la administración de recursos interactivos y pedagógicos efectivos que motiven dicho intercambio, lo que abre espacios a la posibilidad de la figura de usuarios/as intervinientes, aspecto que se traduce en la inclusión de sus experiencias para lograr una identificación con los temas y una cercanía más allá del contenido, 
generando así un sentido de pertenencia a este territorio virtual, tal como se menciona en el análisis de las debilidades del FODA. En términos prácticos, esto significa que el sitio debiera ser un lugar expedito a la hora de posibilitar acciones como agregar, opinar, reflexionar y discutir sobre los datos alojados en él, así como promover la participación en la formulación de objetivos, ya sea ampliándolos o modificándolos más allá de la apreciación subjetiva individual, en otras palabras proyectándolos a lo intersubjetivo.

Lo anterior está en estrecha relación con otras investigaciones recientes en el área, donde a la figura de comunidades de aprendizaje en línea con fines de aprendizaje formal o informal, se le exige funciones para colaborar y cooperar, así como medios para crear y compartir recursos. Los docentes utilizarían esas comunidades para actuar a través de ellas en su propio interés en la aplicación de nuevos métodos educativos. En esa comunidad se debería disponer de un servicio de navegación que permitiera personalizar, compartir y buscar información y recursos relevantes, tal como lo señala Berlanga et al. (2011). Cabe señalar que la versión educativa de algunas de estas redes podría ser considerada como "red de aprendizaje", es decir, como grupos de personas que aprenden juntas ajustando el cuándo, el cómo y el dónde llevan a cabo las actividades o realizan las tareas asignadas de acuerdo con sus necesidades y disponibilidad (Coll y Bustos, 2010).

La inclusión en el diseño e implementación de una plataforma educativa del concepto de informática comunitaria (Gurstein, 2000) es un punto interesante en este contexto, ya que se centra justamente en el estudio interdisciplinario sobre la práctica del diseño, implementación y gestión de las tecnologías de la información y la comunicación desarrolladas por las comunidades para resolver sus propios problemas. Esta disciplina tiene en cuenta la investigación de la ciencia social sobre la repercusión en la sociedad de las tecnologías, conocida también como informática social, así como las técnicas de análisis y diseño de los sistemas de información y comunicación. Es una estrategia o disciplina que combina tecnología y organización social, y que pone en red los esfuerzos comunitarios por el desarrollo socioeconómico. Puede definirse como el estudio sobre las aplicaciones y sus logros en las comunidades para alcanzar objetivos sociales, políticos, económicos y culturales.

Las perspectivas o proyecciones que se originan de los aprendizajes descritos y que tienen relación con la continuidad en el desarrollo de plataformas educativas en Chile, con particular interés en la comunidad, cobran relevancia por varias razones:

A. El interés creciente a nivel gubernamental por incorporar la tecnología en el aula. A partir del año 2011, Internet es parte de un derecho humano necesario para la libertad de expresión de acuerdo a la Organización para la Seguridad y la Cooperación en Europa (OSCE) y la Organización de Naciones Unidas (ONU) (Toudert, 2013). Tomando en cuenta estos antecedentes, el acceso a Internet y disponibilidad del uso de las TIC se ha vuelto un eje central de los diferentes gobiernos. En Chile, Enlaces tiene como misión Integrar las TIC en el sistema escolar para lograr el mejoramiento de los aprendizajes y el desarrollo de competencias digitales en los diferentes actores, preocupado de la reducción de la brecha digital en profesores, cambio en la percepción del rol de las TIC, desarrollo de competencias esenciales, y acceso a través de las escuelas. Por otro lado, el año 2011 se ha incorporado a la agenda nacional el SIMCE TIC para medir habilidades de los estudiantes. Desde el 2012, además, se está implementando "Conectividad para la educación" que aseguraría la conexión para establecimientos subvencionados.

B. Nuevos problemas de desigualdades que se extienden o se generan desde lo tecnológico. Las razones de dicha desigualdad pueden ser muy distintas, en un caso 
puede ser que realmente no existan los medios, y en otros simplemente se ignora o se descalifica a priori. En relación a esto, observando el primer censo de informática educativa, radiografía TIC (CEPPE 2012) de los establecimientos educacionales del país 2012, a nivel nacional el $52 \%$ de las escuelas se encuentra en nivel incipiente, el $22 \%$ en elemental, el $23 \%$ en intermedio, y sólo el $3 \%$ en avanzado. Un hallazgo preocupante es que el $52 \%$ de los 9.260 establecimientos municipales, subvencionados y privados chilenos que participó en la evaluación, no aprovecha la infraestructura informática y de software que tienen para enseñar a sus estudiantes. De acuerdo al estudio internacional TALIS (Teaching and Learning International Survey) de la OCDE, la segunda necesidad de capacitación declarada por los profesores es el uso pedagógico de TIC. En este sentido, si bien los profesores chilenos declaran mayoritariamente saber utilizar las TIC, es probable que exista una enorme necesidad de capacitación en el uso pedagógico de las mismas. Incluso, algunos estudios (Claro, 2010) han planteado la aparición de una "segunda brecha digital" (Claro et al., 2011) (la primera es el acceso), que tiene que ver con la capacidad de aprovechar de manera efectiva las potencialidades que se abren al tener disponibles estas herramientas. Según explican, mientras mejor es el contexto socio-cultural de los alumnos, más desarrollan dichas capacidades. Respecto a las competencias docentes, los profesores que están entrando al sistema educacional cuentan con mejores competencias para utilizar las TIC.

C. Las comunidades se han vuelto algo cotidiano en contextos recreativos (juegos, lecturas, intercambios, amistad, etc.), el entorno educativo no puede quedar fuera de esa forma de interacción social que marca la introducción de los aparatos tecnológicos fuertemente orientados a la superconectividad. Consideramos aquí el concepto de conectivismo de Siemens quien plantea que el conocimiento se basa en el deseo de aprender, pero a través de interacciones entre personas y dispositivos tecnológicos; el establecimiento de redes y de la actualización permanente de la información. El estudiante, por lo tanto, aprende continuamente por medio de redes y por las conexiones que establece. Según esta teoría, el aprendizaje está construido/creado en comunidad y el conocimiento es el resultado de la construcción conjunta de expertos y aprendices (Siemens, 2005, en Martin, 2011).

En un primer momento, el desarrollo e implementación de experiencias educativas en entornos TIC estuvo centrado en las personas y los contenidos. Desde los últimos años, debido a los análisis de las investigaciones científicas, éstas han ido derivando a los entornos comunitarios de aprendizaje; ejemplo de implementación de comunidades de aprendizaje como Colombia Aprende (2008), Centros TIC comunidad de Andalucía, España (2009) plataforma de gestión de recursos, AGORA, México (2011), entre otros, nos indican que, como señala Jameson (1991), la tecnología de la sociedad contemporánea no es hipnótica y fascinante por sí misma; por lo tanto, debemos buscar la forma de aunar y darle un sentido, especialmente desde lo educativo. En otras palabras, se hace necesario tecnología y desarrollo de calidad que se cree desde la comunidad hacia la comunidad recursivamente a favor de la generación de redes, de asociación, de apoyo, de estructura, de conocimientos, de acción.

Las comunidades virtuales se proyectan hoy en día como un espacio comunicativo global. Es un espacio colectivo de reordenamiento de mediaciones simbólicas, como señala Hopenhayn (2003), que permite y motiva reagrupaciones de individuos donde la homogeneidad espacial no cuenta. Tampoco parece requerirse continuidad en el tiempo, con lo cual se alteran categorías básicas de los sujetos como linealidad-continuidad del tiempo y homogeneidad del espacio, que a su vez son categorías básicas de la cultura. Como señala Castells, "este tiempo lineal, irreversible, medible y predecible se está haciendo pedazos en la sociedad red", y lo que adviene 
es la mezcla de tiempos para crear un universo eterno, no autoexpansivo sino autosostenido, no cíclico sino aleatorio, no recurrente sino incurrente [...] las nuevas tecnologías de la información, incorporadas en la nueva sociedad red, facilitan decisivamente [...] la huida de la cultura del reloj" (Castells, 1999, cit. en Hopenhayn, 2003:18).

La construcción del conocimiento se establece gracias al intercambio de diferentes niveles de conocimiento y experiencias de los profesores, quienes se prestan ayuda colaborando mutuamente. El carácter virtual de la comunidad facilita el intercambio y la comunicación entre sus miembros promoviendo los procesos de enseñanza aprendizaje (Coll y Bustos, 2010). Por lo tanto, las innovaciones pedagógicas estarían configuradas por perspectivas donde la información y la interactividad (Cabero et al., 2004) permitan adquirir el "sentido", para que las personas no sean meras receptoras pasivas de información, sino procesadoras activas y conscientes de la misma. La instantaneidad facilita la rapidez al acceso e intercambio de información rompiendo las barreras espacio temporales, lo que permite influenciar más sobre los procesos que sobre los productos y la posibilidad de la interconexión de las mismas. La escuela en el ciberespacio, en este caso, constituye el lugar en que se desarrolla una construcción conjunta, realizada de forma cooperativa de acuerdo a las posibilidades que ofrece la tecnología informática y los intereses, gustos, preferencias [...] de sus creadores y usuarios (Cabero et al., 2004).

En el artículo se ha revisado el contexto en el cual se diseñó e implementó el proyecto PEPE, la descripción general de sus resultados, los aprendizajes y las perspectivas futuras que se deben sustentar en una comunidad de aprendizaje que considera la interacción, participación, compromiso, apropiación e identidad, donde las TIC no son solo una herramienta de la cultura que media el aprendizaje, sino un potencial instrumento de cambio psicológico y comunitario en la medida en que son utilizadas como una herramienta mediadora de los procesos de aprendizaje.

\section{REFERENCIAS BIBLIOGRÁFICAS}

Baudrillard, J. (2007). Cultura y Simulacro. Barcelona: Kairos.

Berlanga, A.; Rusman, E.; Bitter-Rijpkema, M. y Sloep, P. (2011). Redes de Aprendizaje, Aprendizaje en Red. Comunicar, vol. 19, n. 37, 55-238.

Brunner, J. (2007). PISA - 2006: Resultados de Chile. Rescatado el 13 de octubre del 2013 de http://200.6.99.248/ bru487cl/files/2007/12/pisa_2006_prese.html

Cabero, J., Llorente, M. y Román, P. (2004). Las herramientas de comunicación en el aprendizaje mezclado. Píxel-Bit, $n$. 23, 27-41.

Canales, R. y Marques, P. (2007). Factores de buenas prácticas educativas con apoyo de las TIC. Análisis de su presencia en tres centros educativos. Educar, n. 39, 115133.

Canales, R., Quintana, M., Alarcón, C. y otros (2012). “Aprender con tecnologías en la Sociedad del Conocimiento". Osorno: Universidad de Los Lagos.

CEPPE. (2012). Primer censo de Informática Educativa: radiografía TIC de los establecimientos educacionales del país. Centro de Estudios de Políticas y Prácticas en Educación, n. 10. Recuperado el 27 de junio del 2013 en http://www.ceppe.cl/images/stories/recursos/notas/10Notas-para-Educacion-Enero2012.pdf 
Claro, M. et al. (2011). Aporte al sistema educativo a la reducción de las brechas digitales. Una mirada desde las mediciones PISA. CEPAL

Claro, M. (2010). Impacto de las TIC en los aprendizajes de los estudiantes. Estado del Arte. CEPAL

Coll, C. y Bustos, A. (2010). Los entornos virtuales como espacios de enseñanza y aprendizaje. Una perspectiva psicoeducativa para su caracterización y análisis. Revista Mexicana de investigación educativa, vol. 15, n. 44, 163-184.

Enlaces. (2013). Informe final "Sistema de Medición del Desarrollo Digital de los Establecimientos Educacionales". Ministerio de Educación. Santiago. Recuperado el 8 de agosto del 2013 en

http://www.enlaces.cl/tp_enlaces/portales/tpe76eb4809f44/uploadImg/File/2013/doc/ce nso/Censo_de_Informatica_Educativa.pdf

Enlaces. (2012). SIMCE TIC 2011. Ministerio de Educación. Santiago. Recuperado el 27 de junio del 2013 en http://bit.ly/18bQfQo

Gertz, C. (1997). El antropólogo como autor. España: Paidos Ibérica.

Gurstein, M. (2000). Community Informatics: Enabling Communities with Information and Communications Technologies. Hershey USA, London UK: Idea Group Publishing.

Hopenhayn, M. (2003) Informes y estudios especiales: Educación, comunicación y cultura en la sociedad de la información: una perspectiva latinoamericana. Naciones Unidas, Santiago de Chile. Recuperado 15 de junio del 2013 en http://www.eclac.org/publicaciones/xml/8/11678/lcl1844e.pdf

Jameson, F. (1991). El postmodernismo o la lógica cultural del capitalismo avanzado. Barcelona: Paidós.

Martín, O. (2011). Educar en comunidad: promesas y realidades de la Web 2.0 para la innovación pedagógica en Carneiro. En R. Toscazo, J. Díaz, Los desafíos de las TIC para el cambio educativo. (pp. 79-93). EEUU: Organización de los Estados Iberoamericanos, Fundación Santillana.

Mineduc. (2008). Resultados Nacional SIMCE 2007. Santiago de Chile: Ministerio de Educación. Recuperado 13 de octubre del 2013 en http://www.agenciaeducacion.cl/wpcontent/files_mf/informenacionalderesultadossimce20072.5m.pdf

Mineduc. (2011). Anuario estadístico de la evaluación docente 2011. CPEIP. Recuperado el 9 de agosto del 2013 en http://bit.ly/18bT83W.

Peña, P. (2002). El diario de la Sociedad Civil: Usos y apropiaciones por parte de la de la comunidad de organizaciones emisoras miembros del sitio web. Recuperado el 1 de $\quad$ agosto del 2013 en http://www.periodismo.uchile.cl/encuentroconosur/ponencias/2/2d_patriciapena.h $\underline{\operatorname{tml}}$

PNUD (2006). Desarrollo humano en Chile. Las nuevas tecnologías un salto al futuro. Santiago de Chile: Programa de las Naciones Unidas para el Desarrollo.

Rinaldi, M. (2011). Revolución Mobile Learning. España: Printing History

Vieluf, S. Kaplan, D. Klieme, E. Bayer, S. (2012) Teaching Practices and Pedagogical Innovation Evidence from TALIS. Paris: OECD.

Toudert, D. (2013). La brecha digital en los contextos de marginación socioterritorial en localidades mexicanas: exploración y discusión. Comunicación y Sociedad, n.19, 153-180.

Valenzuela, J.P., Bellei, C. y De los Ríos, D. 2010. Segregación escolar en Chile. En S. Martinic y G. Elacqua, eds., ¿Fin de Ciclo? Cambios en la gobernabilidad del 
sistema educativo. Santiago de Chile: OREALC-UNESCO- Pontificia Universidad Católica de Chile.

Vizer, E. (2003). La trama (in)visible de la vida social: comunicación, sentido y realidad. Buenos Aires: La Crujía ediciones. 